\title{
Aspectos reprodutivos de Potimirim glabra (Kingsley) (Crustacea, Decapoda, Atyidae) no Rio Sahy, Mangaratiba, Rio de Janeiro, Brasil
}

\author{
Giovana Valverde Lima ${ }^{1}$ \\ Lídia Miyako Yoshii Oshiro ${ }^{1}$
}

\begin{abstract}
The reproductive aspects of the freshwater shrimp Potimirim glabra (Kingsley) (Crustacea, Decapoda, Atyidae) from Sahy River, Mangaratiba, Rio de Janeiro, Brazil. The reproductive aspects of the freshwater shrimp Potimirim glabra (Kingsley, 1878) were studied from September 1997 to February 1999. Monthly, samples were collected with sieves, on the river edge, under the edging vegetation or in small pools. At the laboratory, shrimps were sexed and measured in their total lenght, from the tip of the rostrum to the end of telson with precision callipers. The ovigerous shrimps were separated and counted for fecundity determination. The eggs diameter was measured with calibrated stereomicroscope. A total of 3281 shrimps were collected, among which, $47 \%$ male, $46 \%$ female $(13.4 \%$ ovigerous females) and $7 \%$ young shrimps. The Sex ratio was 1:1. Ovigerous females were present during all seasons, but the reproductive peak occurred in spring. The ovigerous female showed total lenght from 9.5 to $26.0 \mathrm{~mm}$ and carapace lenght from 1.6 to 7.3 $\mathrm{mm}$. The mean fecundity was $545 \mathrm{eggs}$ per female, with a minimun of 223 eggs and a maximum of 860 eggs. The first sexual maturation probably occur at $9.5 \mathrm{~mm}$ total lenght. The mean diameter of the eggs size was $0.32 \mathrm{~mm}$ (minor) and $0.53 \mathrm{~mm}$ (major). KEY WORDS. Crustacea, Decapoda, Atyidae, Freshwater shrimp, Potimirim glabra, Reproduction, Sahy River, Rio de Janeiro State
\end{abstract}

Segundo BarRos \& Fontoura (1996b), o gênero Potimirim Holthuis, 1954 pertence à família Atyidae, estando representada atualmente por cinco espécies: $P$. mexicana De Saussure, 1857, P. brasiliana Villalobos, 1959, P. americana (Guérin-Méneville, 1855), $P$. potimirim (Müller, 1881) e $P$. glabra (Kingsley, 1878), todas registradas apenas para as Américas.

De acordo com RAMOS-PORTO \& COELHO (1998), no Brasil, existem apenas duas espécies representantes deste gênero: $P$. potimirim (Müller, 1881) e $P$. glabra (Kingsley, 1878). P. brasiliana foi sugerido como sinonímia de P. glabra (SMALLEY 1963; CHACE \& HoBBS 1969), mas, posteriormente MANNING \& HoBBS (1977) reconheceram a validade da espécie proposta por VILLALOBOS (1959). HOLTHUIS (1986) achou justificável esta proposta de sinonímia, mas sugeriu a análise de maior quantidade de material, para corroborar esta hipótese.

Potimirim glabra ocorre em El Salvador, Nicarágua, Costa Rica, Panamá, Venezuela, Colômbia, Dominica e Brasil (Rodriguez 1980; HolthuIS 1986). No Brasil, foi registrado nos Estados do Rio de Janeiro, São Paulo e Santa Catarina (RAMOS-PORTO \& COELHO 1998).

1) Estação de Biologia Marinha, Universidade Federal Rural do Rio de Janeiro. Rua Sereder, Itacuruçá, 23860-000 Mangaratiba, Rio de Janeiro, Brasil. E-mail: oshiro@ ufrrj.br 
Em relação a $P$. brasiliana, VILLALOBOS (1959) relatou a distribuição geográfica e a ecologia, e fez considerações sistemáticas. Enquanto, BARROS \& FONTOURA (1996a,b) estudaram em Santa Catarina a reprodução e o crescimento de $P$. glabra.

O presente trabalho visa contribuir para o conhecimento da biologia de $P$. glabra, determinando-se a época de reprodução, o tamanho da primeira maturação sexual e populacional, a fecundidade e o tamanho médio dos ovos da população que habita o Rio Sahy, Município de Mangaratiba, Rio de Janeiro.

\section{MATERIAL \& MÉTODOS}

Potimirim glabra foi coletado mensalmente no Rio Sahy, Município de Mangaratiba, Rio de Janeiro ( $22^{\circ} 56$ 'S; $44^{\circ} 01^{\prime} \mathrm{W}$ ), durante o período de setembro/1997 a fevereiro/1999.

O Rio Sahy está inserido dentro dos limites da fazenda Santa Helena, desembocando na Praia do Sahy e sua nascente está localizada na região da Serra do Piloto, no bairro do Rubião. Foram pré-estabelecidas seis estações de coletas segundo a distância em relação à foz e ao tipo de declividade do terreno, sendo que as estações V e VI sofrem constante influência da salinidade.

Todos os exemplares de camarão foram capturados com peneiras de $0,1 \mathrm{~mm}$ de abertura de malha, passadas sob a vegetação marginal e substratos depositados sobre o fundo do rio. Durante o estudo foram registrados dados de temperatura do ar e da água, com termômetro de mercúrio, salinidade com um salinômetro e a profundidade com uma régua graduada.

Os camarões coletados foram levados ao laboratório da Estação de Biologia Marinha, Universidade Federal Rural do Rio de Janeiro (UFRRJ) em Itacuruçá, fixados em formaldeído a $10 \%$, sendo posteriormente conservados em álcool a $70 \%$ e etiquetados.

Os camarões foram identificados, separados por sexo e medidos o comprimento da carapaça (medida entre a órbita ocular e o bordo posterior dorsal da carapaça) e o comprimento total (medida entre a extremidade distal do rostro e a extremidade posterior do telso), utilizando-se um paquímetro de $0,1 \mathrm{~mm}$ de precisão.

O período reprodutivo foi determinado para a espécie através da análise das frequiências relativas de fêmeas ovígeras, capturadas ao longo dos meses de coleta.

A maturidade sexual individual foi determinada pelo comprimento total apresentado pela menor fêmea ovígera capturada, enquanto a estimativa da maturidade sexual populacional foi realizada determinando-se a classe de comprimento total correspondente à frequêencia de 50\% do total de fêmeas ovígeras (VAZZOLER 1981).

A fecundidade média de $P$. glabra foi determinada pela retirada e contagem do total do número de ovos aderidos aos pleópodos de 50 fêmeas ovígeras. Os ovos foram retirados com auxílio de pinças e estiletes, colocados numa placa quadriculada e contados com auxílio de um contador manual sob um microscópio estereoscópico.

A relação entre a fecundidade e o comprimento total, assim como a fecundidade e o comprimento do cefalotórax, foi analisada através da regressão linear descrita pela equação $Y=b x+a$. 
O tamanho dos ovos foi obtido através da medida do diâmetro maior e do menor, tomando-se uma amostra de 20 ovos por fêmea. Esses ovos foram medidos, em aumento de $25 \mathrm{x}$, com auxílio de uma ocular micrométrica do microscópio estereoscópico.

\section{RESULTADOS E DISCUSSÃO}

A temperatura do ar nos locais de coleta variou de 15 a $31^{\circ} \mathrm{C}$, enquanto a temperatura da água, de 13 a $25^{\circ} \mathrm{C}$. Os valores observados tanto para a temperatura do ar quanto da água, apresentaram os menores e maiores valores em junho e janeiro, respectivamente. A salinidade variou de 0 a $5 \%$ e a profundidade não ultrapassou a $80 \mathrm{~cm}$, sendo verificada uma média anual em torno de $32 \mathrm{~cm}$, com amplitude de variação de 18 a $79 \mathrm{~cm}$.

Foi capturado um total de 3.281 indivíduos, sendo 1.517 fêmeas (46,2\%), 1.530 machos $(46,6 \%)$ e 234 juvenis $(7,1 \%)$. Das fêmeas capturadas, 204 indivíduos eram ovígeras, representando $13,4 \%$.

Potimirim glabra apresentou fêmeas ovígeras durante todo o ano de estudo, com maior freqüência relativa durante a primavera e redução gradativa até o inverno (Fig. 1).

BARROS \& FONTOURA (1996a) encontraram o período reprodutivo da espécie, em Santa Catarina, ocorrendo marcadamente na primavera-verão e parte do outono, com pico reprodutivo ocorrendo de janeiro a julho.

MÜLLER (1892) e BARRos \& FONTOURA (1996a), ambos para a região de Santa Catarina, também, encontraram reprodução do tipo periódica para a espécie. No entanto, SMALLEY (1963) encontrou fêmeas ovígeras de P. brasiliana durante todo o período de estudo na Costa Rica.

De acordo com LiMA \& OSHIRO (1999), o período reprodutivo de $P$. potimirim também ocorreu durante a primavera-verão, neste mesmo local. Mas, observaram a ausência das fêmeas ovígeras durante o outono e o inverno.

O comprimento total das fêmeas ovígeras de $P$. glabra variou de 9,5 a 24,0 $\mathrm{mm}$, correspondendo à classe de comprimento do cefalotórax de 1,6 a 7,3 $\mathrm{mm}$. $\mathrm{O}$ tamanho da menor fêmea ovígera foi de $9,5 \mathrm{~mm}$ de comprimento total e 3,0 mm de comprimento do cefalotórax.

A maturidade sexual populacional foi estimada para a classe de 16,1 a 18,0 mm de comprimento total (3,0 a 6,0 mm de comprimento do cefalotórax) (Fig. 2). Mas, BARRos \& FonTOURA (1996a), encontraram para P. glabra em Santa Catarina, a maturidade sexual populacional na classe de comprimento total de 21,7 a $22,4 \mathrm{~mm}(6,2$ a $6,4 \mathrm{~mm}$ de comprimento do cefalotórax). Portanto, a maturidade sexual foi atingida em tamanhos maiores aos observados para a população no Rio Sahy, isso provavelmente, devido as diferenças em relação as latitudes das áreas estudadas.

O número médio de ovos carregados por fêmeas de $P$. glabra foi de $545 \pm$ 166 ovos, com uma variação de 223 a 860 ovos, de acordo com o comprimento total desses indivíduos. Verificou-se que a classe de maior freqüência de fêmeas ovígeras foi daquelas carregando de 501 a 600 ovos nos pleópodos (Fig. 3). 

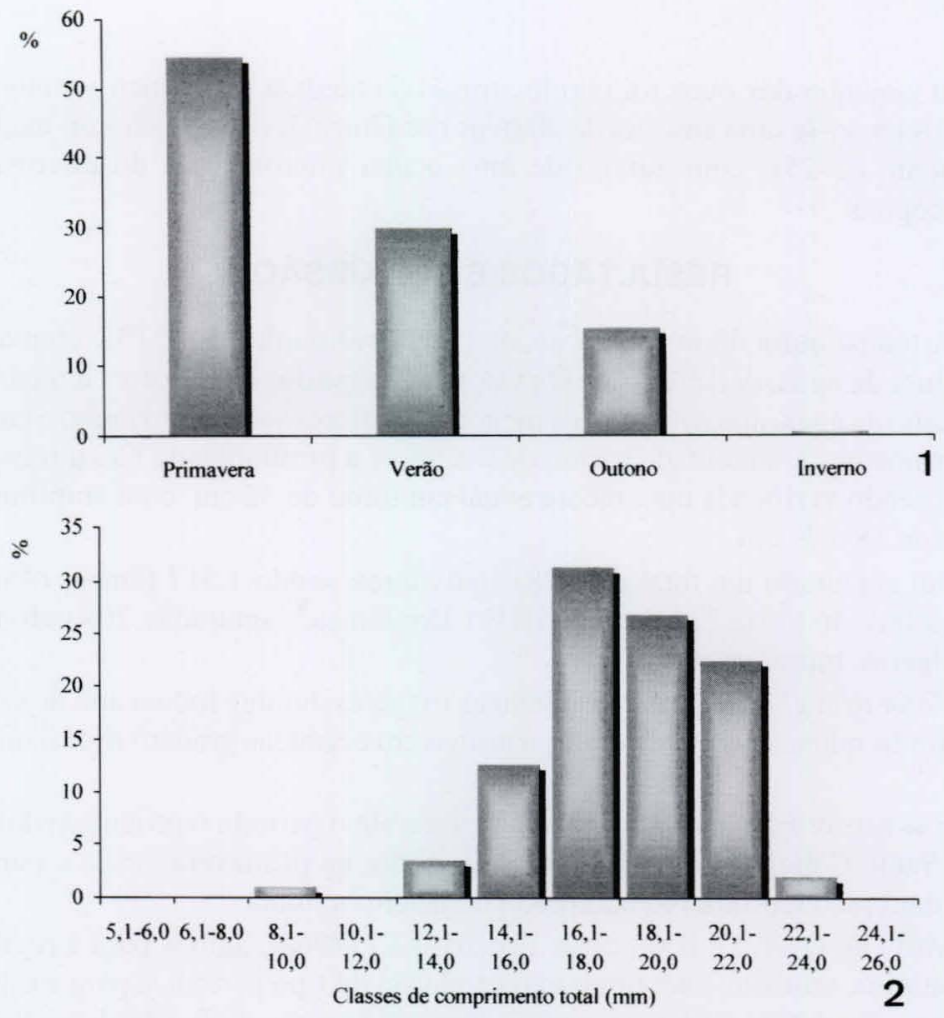

Figs 1-2. Fêmeas ovígeras de Potimirim glabra no Rio Sahy, durante o período de setembro/1997 a fevereiro/1999: (1) distribuição sazonal; (2) distribuição da freqüência relativa nas classes de comprimento total $(\mathrm{mm})$.

BARROS \& FONTOURA (1996a) encontraram valores bem superiores de fecundidade para esta espécie, com uma média de 896 ovos, com variação de 389 a 1541 ovos dispostos nos pleópodos.

A relação entre o número de ovos carregados pelas fêmeas e o comprimento do cefalotórax foi descrita pela equação linear $Y=114,98+112,39 x\left(r^{2}=0,27\right)$, devido ao seu melhor ajuste (Fig. 4). Verificou-se um baixo coeficiente de determinação, mas observa-se que o número de ovos têm uma tendência a uma relação direta com o tamanho do indivíduo, assim como nos demais crustáceos decápodos (Tab. I).

O comprimento dos ovos variou de 0,28 a $1,0 \mathrm{~mm}$, enquanto a largura, de 0,40 a $0,68 \mathrm{~mm}$, com valores médios de $0,32 \pm 0,13$ e $0,53 \pm 0,08 \mathrm{~mm}$, respectivamente. Os ovos analisados apresentaram forma elíptica, observando-se na fase inicial de desenvolvimento, coloração alaranjada e ausência de manchas ocelares, enquanto na fase final verificou-se coloração marrom, com manchas ocelares bem definidas.

DAVIS (1963), verificou que os ovos de P. glabra próximos ao período de postura apresentam tamanho médio de $500 \times 300 \mu \mathrm{m}$. 

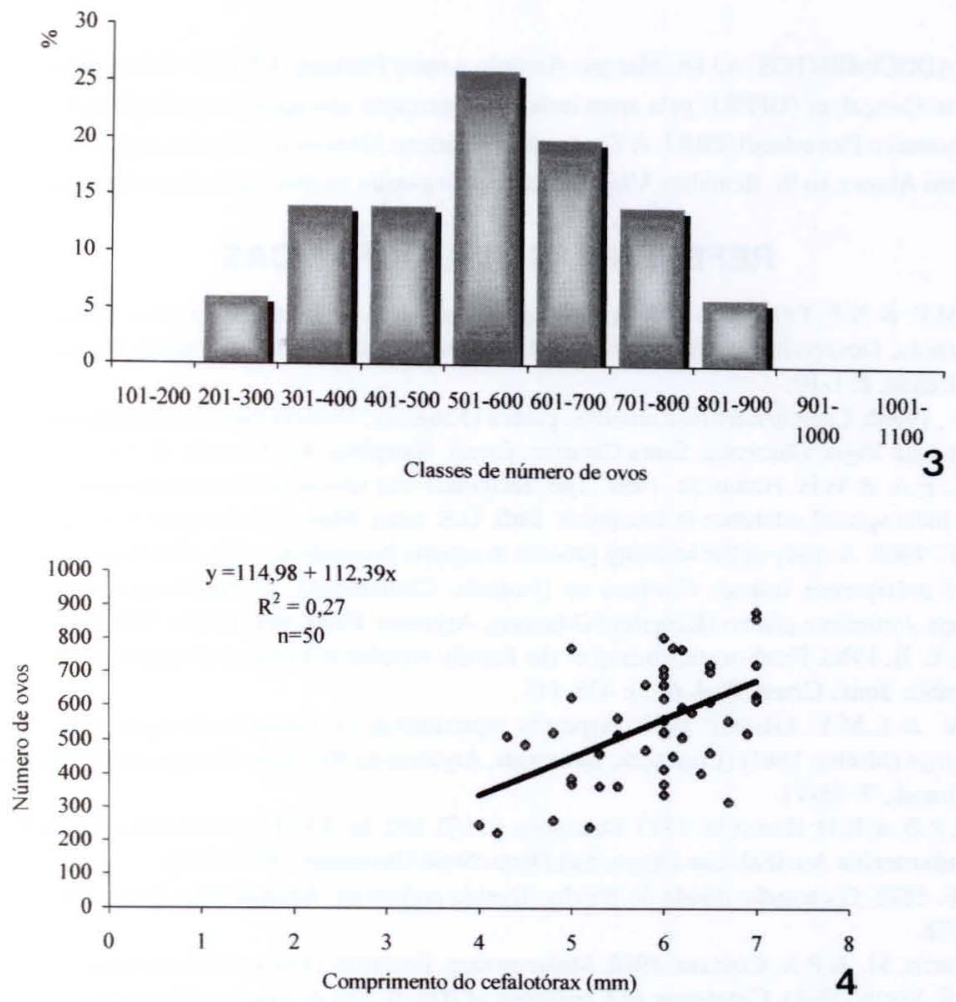

Figs 3-4. Potimirim glabra no Rio Sahy, durante o período de setembro/1997 a fevereiro/1999: (3) distribuição da freqüência relativa de fêmeas ovígeras nas classes de número de ovos; (4) relação entre o número de ovos e o comprimento do cefalotórax das fêmeas ovígeras $(\mathrm{mm})$.

Tabela I. Distribuição do número de fêmeas ovígeras e o número médio de ovos de $P$. glabra nas classes de comprimento total $(\mathrm{mm})$, no Rio Sahy, durante o período de setembro/1997 a fevereiro/1999.

\begin{tabular}{ccc}
\hline Classes de comprimento total $(\mathrm{mm})$ & Número de fêmeas ovigeras & Número médio de ovos \\
\hline $8,1-10,0$ & 0 & 0 \\
$10,1-12,0$ & 0 & 0 \\
$12,1-13,0$ & 0 & 0 \\
$14,1-16,0$ & 1 & 261 \\
$16,1-18,0$ & 5 & 442 \\
$18,1-20,0$ & 16 & 505 \\
$20,1-22,0$ & 25 & 592 \\
$22,1-24,0$ & 3 & 638 \\
\hline
\end{tabular}

Segundo LIMA \& OSHIRO (1999), os ovos de $P$. potimirim apresentam forma elíptica, porém com tamanho médio de $450 \mu \mathrm{m}$ de comprimento e $300 \mu \mathrm{m}$ de largura, verificando-se, que os ovos de $P$. glabra eram ligeiramente maiores que os de $P$. potimirim. 
AGRADECIMENTOS. Ao Dr. Marcelo Antônio Amaro Pinheiro (UNESP -Jaboticabal) e Dr. Lenício Gonçalves (UFFRJ) pela apreciação da dissertação apresentada ao Curso de Ciências Ambientais e Florestais/UFRRJ. À Zootecnista Charlene Moreira da Silveira, ao Sr. Casemiro Antônio Alves e ao Sr. Benedito Albernaz Lima pelo auxílio na realização das coletas mensais.

\section{REFERÊNCIAS BIBLIOGRÁFICAS}

Barros, M.P. \& N.F. Fontoura. 1996a. Biologia reprodutiva de Potimirim glabra (Kingsley, 1878) (Crustacea, Decapoda, Atyidae), na Praia da Vigia, Garopaba, Santa Catarina, Brasil. Nauplius, Rio Grande, 4: 1-10.

-1996b. Crescimento de Potimirim glabra (Kingsley, 1878) (Crustacea, Decapoda, Atyidae), na Praia da Vigia, Garopaba, Santa Catarina, Brasil. Nauplius, Rio Grande, 4: 11-28.

ChACE JR., F.A. \& H.H. HobBS JR. 1969. The freshwater and terrestrial Decapoda crustaceans of the West Indie special reference to Dominica. Bull. U.S. natn. Mus., Washington, D.C., 292: 1-243.

DAVIS, C.C. 1963. A study of the hatching process in aquatic invertebrates. IX. Hatching with the brood sac of oviviparous isopod, Cirolana sp (Isopoda, Cirolanidae). X. Hatching in the freshwater shrimps, Potimirim glabra (Kingsley) (Macrura, Atyidae). Pacif. Sci. 18 (4): 378-384.

HolthuIs, L. B. 1986. Fresh-water shrimp of the Family Atyidae (Crustacea:Decapoda) from Western Colombia. Jour. Crust. Biol. 6 (3): 438-445.

Lima, G.V. \& L.M.Y. Oshiro. 1999. Aspectos reprodutivos de camarão de água doce Potimirim potimirim (Muller, 1881) (Crustacea, Decapoda, Atyidae) do Rio Sahy, Mangaratiba/RJ. Nauplius, Rio Grande, 7: 65-71.

Manning, R.B. \& H.H. HobBs JR. 1977. Decapoda, p. 157-162. In: S.H. Hulbert (Ed.). Biota Aquatica da Sudamerica Austral. San Diego, San Diego State University, XIV+342p.

MülLer, F. 1892. O camarão miúdo do Itajahy Atyoida potimirim. Archos Mus. Nac., Rio Janeiro, 8: 155-178.

Ramos-Porto, M. \& P.A. Coelho. 1998. Malacostraca. Eucarida (Alpheoidea excluded), p. 325-350. In: P.S. Young (Ed.). Catalogue of Crustacea of Brazil. Rio de Janeiro, Museu Nacional, 717p.

Rodriguez, G. 1980. Los crustaceos decapoda de Venezuela. Caracas, Instituto Venezoelano de Investigaciones Científicas, 493p.

Smalley, A.L. 1963. The genus Potimirim in Central America (Crustacea, Atyidae). Revta Biol. Trop. 11 (2): 117-183.

VAZzoler, A.E.A.M. 1981. Manual de métodos para estudos biológicos de populações de peixes: reprodução e crescimento. Brasília, CNPq, Programa Nacional de Zoologia, 106p.

Villalobos, F.A. 1959. Contributión al conocimiento de los Atyidae de México. II (Crustacea, Decapoda). Estudio de algunas especies del genero Potimirim (=Ortmannia), com descripcion de uma especie nueva en Brasil. An. Inst. Biol. Univ. Méx. 30 (1-2): 269-330.

Recebido em 09.XI.2001; aceito em 03.IX.2002. 